\title{
Some Common SNPs of the T-Cell Homeostasis-Related Genes Are Associated with Multiple Sclerosis, but Not with the Clinical Manifestations of the Disease, in the Polish Population
}

\author{
Monika Choraży $\mathbb{D D}^{1}{ }^{1}$ Natalia Wawrusiewicz-Kurylonek $\left(\mathbb{D},{ }^{2,3}\right.$ Edyta Adamska-Patruno ${ }^{(\mathbb{D}},{ }^{4}$ \\ Olga Zajkowska $₫,{ }^{5}$ Katarzyna Kapica-Topczewska $₫{ }^{1},{ }^{1}$ Renata Posmyk $\left({ }^{1},{ }^{3}\right.$ \\ Adam Jacek Krętowski $\oplus^{2,4}{ }^{2,4}$ Jan Kochanowicz, ${ }^{1}$ and Alina Kułakowska $\oplus^{1}$ \\ ${ }^{1}$ Department of Neurology, Medical University of Bialystok, M. Sklodowskiej-Curie 24A, 15-276 Bialystok, Poland \\ ${ }^{2}$ Department of Endocrinology, Diabetology and Internal Medicine, Medical University of Bialystok, M. Sklodowskiej-Curie 24A, \\ 15-276 Bialystok, Poland \\ ${ }^{3}$ Department of Clinical Genetics, Medical University of Bialystok, Waszyngtona 13 15-089 Bialystok, Poland \\ ${ }^{4}$ Clinical Research Centre, Medical University of Bialystok, M. Sklodowskiej-Curie 24A, 15-276 Bialystok, Poland \\ ${ }^{5}$ Faculty of Economic Sciences, University of Warsaw, Dluga 44/50, 00-241 Warsaw, Poland
}

Correspondence should be addressed to Monika Chorąży; chorazym@op.pl

Received 9 September 2020; Revised 4 November 2020; Accepted 5 November 2020; Published 11 November 2020

Academic Editor: Samanta Taurone

Copyright (C) 2020 Monika Chorąży et al. This is an open access article distributed under the Creative Commons Attribution License, which permits unrestricted use, distribution, and reproduction in any medium, provided the original work is properly cited.

Purpose. Multiple sclerosis (MS) is an autoimmune disease, and genetic factors play an important role in its pathogenesis and progression. The aim of our study was to evaluate the frequencies of alleles and genetic variants of the T-cell homeostasis-related genes, in subjects with MS, as well as to investigate the association with MS clinical manifestations and disability. Methods. 94 subjects with MS and 160 healthy individuals have been genotyped for seven common single-nucleotide variants in IL-2RA, CTLA4, CD40, and PADI4 genes. The ages of onset, duration of the disease, and clinical condition of the MS subjects were analysed. We used the $\mathrm{Chi}^{2}$ test confirmed with Fisher's exact test for statistical analysis. Results. The frequency of allele $\mathrm{T}$ and CT/TT genotypes (rs7093069) in the IL2RA gene, as well as the T allele and CT/TT genotypes in rs12722598, were significantly higher in the control group. The significant differences between studied groups we also found for the G allele and GG/GA genotypes of rs3087243 in CTLA4 gene, which were more common among the control group. The heterozygous genotype TC (rs1883832) of CD40 gene was more common in the control subjects, and the frequency of the alleles and genotypes in the rs1748033 of the PADI4 gene did not differ between the studied groups. Between the studied genotypes, we did not observe any significant differences in the age of onset and duration of disease, including sex stratification. Conclusion. Our results highlight the protective role of some of the T-cell homeostasis-related genetic variants in MS development, but not in its clinical manifestation.

\section{Introduction}

The immunological tolerance balance disturbances, including the presence of specific autoantibodies and the loss of function of some organs, negatively affects the health of patients with autoimmune diseases (ADs) [1]. ADs are complex disorders showing a high frequency of occurrence in the global population $(\sim 3-5 \%)$, and therefore, they are of special interest for the medical scientific community. Knowledge advancement of genetic and environmental components of the etiology of ADs may lead to establishing the basis for optimal prophylaxis, early diagnosis, and perhaps even the choice of effective treatment $[2,3]$. Many GWAS studies (genome-wide association studies) provide from time to time new information about next single-nucleotide variants (SNVs), which may be the molecular risk factors or may have a protective effect for $\mathrm{AD}$ development and progression. These studies are based on multipopulation and multiethnic analyses of genetic variants localised in 
multiple loci, and they are a standard tool for molecular characterisation of the genetic background of complex diseases $[3,4]$. Some of the first-described SNVs are variants within the HLA (human leukocyte antigen) region located on the short arm of chromosome 6 (6p21), which is the molecular background of many autoimmune diseases [5-7]. For example, HLA alleles of class II DRB1 $* 1501-\mathrm{DQB} 1 * 0201$ are associated with an increased risk of multiple sclerosis (MS) development, whereas $\mathrm{HLA}-\mathrm{DRB} 1 * 0301-\mathrm{DQB} 1 * 0201$ is associated with type-1 diabetes mellitus (T1DM) $[8,9]$. GWAS studies have also identified a number of genetic variants among non-HLA genes that present a significant impact on increasing $\mathrm{AD}$ risk, including CTLA-4 (cytotoxic T-lymphocyte-associated antigen 4), IL-2RA (interleukin 2 receptor $\alpha$ ), and PTPN22 (protein tyrosine phosphatase, nonreceptor-type, 22) genes [10-13]. These candidate genes and their allelic variants are related also to T-cell activation and therefore may be involved in the pathogenesis of MS, a common demyelinating neurological disease triggered by environmental factors in genetic risk carriers $[14,15]$. The CTLA-4 is a major negative regulator of T-cell responses [16], and some allelic variations in the CTLA-4 gene show the functional effects on T-cell signalling [17]. IL-2R signalling is known to be essential for regulatory T-cells, and binding IL-2 to its receptor alpha (IL-2RA) on the surface of T-lymphocytes triggers many intracellular signals, resulting in the activation and proliferation of resting T-cells, and in the generation of helper, suppressor, and cytotoxic T-cells, which are immune mediators and induce immune responses $[18,19]$. Some of the single-nucleotide polymorphisms (SNPs) in or near the IL2RA gene have been already associated with the increased risk of several immunemediated diseases, including MS [20]. CD40 is a T-cell receptor costimulator on T-cells and may make these cells effective antigen-resenting cells by upregulating cell surface molecules, which is pivotal for autoimmune disease development [21]. Further, SNPs in the CD40 gene have been linked to MS risk [22], as well as to MS progression and early disease onset in the Novosibirsk-region population [23]. Also, PADI4 was found to be extensively expressed in T-cells [24] and may affect their function [25]. Therefore, the studies investigating associations of genes that we have mentioned above with the risk of MS, considering also possible differences between populations, as well as an influence of other environmental factors that may affect and interact with a genetic background of autoimmune diseases, are of high importance. Because these genes are related to T-cell homeostasis, and MS has been described as a T-cell-mediated demyelinating inflammatory autoimmune disorder [26], we hypothesise that some of the SNVs in or near them may be related to MS development, as well as its clinical manifestations in the Polish population. Considering the above, the aim of our study was to analyse the frequencies of selected SNVs in the Polish population with MS, and their correlations with some of the clinical parameters of the disease.

\section{Materials and Methods}

2.1. Ethics. The study has been approved by the Bioethics Committee (Medical University of Bialystok, R-I002/334/2018) and conducted in accordance with the Hel- sinki Declaration. All participants have signed informed consent forms.

2.2. Participants. Molecular studies were conducted in a group of 94 subjects with MS (47 males and 47 females, mean age $41.15 \pm 9.74 \mathrm{y}$, Table 1 ), which has been previously described in detail [27, 28]. The control group consisted 160 unrelated healthy adults ( 85 males, 75 females; mean age $37.6 \pm 2.16 y$ ) with no family history of autoimmune diseases. Participants from both groups were recruited from the same geographical area and ethnic group.

2.3. Clinical Condition. The ages of onset and duration of the disease were analysed. The clinical condition of MS subjects was evaluated using Kurtzke's Expanded Disability Status Scale (EDSS), at the time of diagnosis, before any treatment was provided.

\subsection{Genetic Analysis}

2.4.1. Genomic DNA Extraction and SNV Analysis. Genomic DNA was isolated with the Qiagen column separation method (QIAamp DNA Blood Mini Kit, Qiagen, Germany) according to the manufacturer's protocols, and then the purity and concentration of the obtained preparations were evaluated by spectrophotometric method using the NanoDrop 2000 device (Thermo Fisher Scientific, USA). All DNA samples were normalised to $50 \mathrm{ng} / \mu \mathrm{l}$. The SNV variants were tested in duplicates using the QuantStudio $12 \mathrm{~K}$ Flex platform on OpenArray plates (Thermo Fisher Scientific, USA). This technology uses TaqMan molecular probes, which are applied to plates and the Genotyping Master Mix (Thermo Fisher Scientific, USA). We analysed seven SNVs in four genes: IL-2RA (rs7093069 and rs12722598), CTLA4 (rs3087243, rs231775, and rs5742906), CD40 (rs1883832), and PADI4 (rs1748033). The list of all investigated variants is presented in Table 2. We used a sample without a template as a negative control to measure any false positive signal caused by molecular contamination. The initial analysis of real-time PCR data was performed with the TaqMan Genotyping Data Analysis Software-Genotyper (Thermo Fisher Scientific).

2.5. Statistical Analysis. For all calculations, the Stata 15 software was used. Since our sample was small, for all the comparisons, we used the $\mathrm{Chi}^{2}$ test confirmed with Fisher's exact test to confirm the results. If possible, we reported the $p$ value and the odds ratios (OR). $p$ value below 0.05 was considered statistically significant. The Hardy-Weinberg equilibrium tests were performed for all allele frequency calculations.

\section{Results}

3.1. Associations between MS and Genetic Variants of the IL2RA Gene. The frequency of the polymorphic T allele of rs7093069 was significantly higher $(p=0.0053)$ in the healthy control group compared to subjects with MS (Table 2). The OR value of 0.56 indicates a strong protective effect of allele T. Similarly, the distributions of the CT and TT genotypes 
TABLE 1: The clinical characteristic of MS subject group.

\begin{tabular}{lccc}
\hline Characteristics & $\begin{array}{c}\text { MS subjects, } \\
n=94\end{array}$ & $\begin{array}{c}\text { Women, } \\
n=47\end{array}$ & $\begin{array}{c}\text { Men, } \\
n=47\end{array}$ \\
\hline $\begin{array}{l}\text { Age at onset } \\
\text { (years) }\end{array}$ & $41.14 \pm 0.79$ & $42.78 \pm 0.98$ & $37.14 \pm 1.11$ \\
$\begin{array}{l}\text { Disease duration } \\
\text { years) }\end{array}$ & $8.12 \pm 0.42$ & $8.18 \pm 0.51$ & $7.96 \pm 0.75$ \\
EDSS & $1.85 \pm 0.10$ & $1.84 \pm 0.12$ & $1.87 \pm 0.19$ \\
\hline
\end{tabular}

Data is presented as mean \pm SD. MS, multiple sclerosis; EDSS, Expanded Disability Status Scale.

in the control group were significantly higher compared to participants with MS $(51.88 \%$ vs. $38.8 \%$, respectively, $p=$ $0.016, \mathrm{OR}=0.52$; and $5.0 \%$ vs. $1.06 \%$, respectively, $p=$ $0.047, \mathrm{OR}=0.15$, Table 2).

We also observed a protective effect of allele $\mathrm{T}$ in rs12722598 (Table 2). The frequency of allele $\mathrm{T}$ was significantly higher in the control healthy group than in the group of individuals with MS (315 vs. 174 individuals, respectively, $p=001, \mathrm{OR}=0.2$ ).

3.2. Associations between $M S$ and Genetic Variants of the CTLA4 Gene. Of the three polymorphisms tested in the CTLA4 gene, our study showed significant differences in allele and genotype frequencies only for rs3087243. The frequency of allele $\mathrm{G}$ was higher in the group of healthy control subjects compared to the group of individuals with MS $(p=0.00, \mathrm{OR}=0.09)$. Similarly, a genotype distribution analysis showed significant differences in the frequency of GG and GA genotypes in the healthy control group compared to the MS group (Table 2). The GG genotype was present only in the group of healthy individuals, and $\mathrm{OR}=0.0038$ indicates an extremely strong protective effect of this genotype. The protective effect was observed for the heterozygous GA genotype, for which we have observed a significantly higher frequency in the healthy control group, compared to the group of subjects with MS (48.12\% vs. $31.91 \%$, respectively, $p=0.00, \mathrm{OR}=0.087$ ).

3.3. Associations between MS and Genetic Variants of the CD40 Gene. > Our analysis of the rs1883832 polymorphisms in the CD40 gene did not show any significant differences in the homozygous genotypes and allele frequencies between the studied groups. We only observed that healthy control individuals carried the heterozygous TC genotype significantly more often than the subjects with diagnosed with MS (59 vs. 23 individuals, respectively, $p=0.00477, \mathrm{OR}=0.55$ ).

3.4. Associations between MS and Genetic Variants of the PADI4 Gene. We did not observe any significant differences between the studied groups in the frequencies of alleles and the genotypes of rs1748033 in the PADI4 gene (Table 2).

3.5. Associations between Investigated Genetic Variants and MS Clinical Manifestations. The analysis of allele and genotype frequency distribution within MS subjects did not show any significant differences between men and women (data not shown). We did not notice any associations between the studied variants, and the age of onset, the duration of the disease, or the disability status (data not shown), including sex stratification.

\section{Discussion}

The rapid technology development and novel models of collaboration between research groups have enabled to discover several genetic risk factors that have been associated with MS. However, due to the fact that the heredity of the disease has not fully been determined yet, the researchers are currently focusing on identifying alleles and genotypes, related pathways, epigenetic mechanisms, and gene-environment interactions, which may have an impact on MS development and progress [29].

In our study, we found that a $\mathrm{T}$ allele of the rs7093069 in the IL2RA gene was significantly more often carried by the healthy control individuals than by participants with MS, which suggests that it may have a protective effect on MS development. Also, the CT and TT genotypes distribution was significantly higher in the healthy control group subjects than in individuals diagnosed with MS, which indicates that carrying the $\mathrm{T}$ allele may have a strong significant protective effect against MS development. The IL2RA gene has been associated with differences in immune cell populations in subjects with MS, and Hartmann et al. found that individuals carrying the IL2RA risk alleles present a significantly increased propensity of $\mathrm{TH}$ ( $\mathrm{T}$ helper) cells to develop into GM-CSF- (granulocyte-macrophage colony-stimulating factor-) producing memory TH-cells and linked this genetic risk factor with a functional feature of TH-cells in MS [30]. The immunologically relevant genes, their target proteins, and T-helper-cell differentiation have been implicated in the pathogenesis of multiple sclerosis in the European population by a collaborative GWAS of 23 research groups working in 15 different countries [31]. As investigated in our study, rs7093069 in the IL2RA gene has already been associated with some ADs, but to the best of our knowledge, our study is the first to report the association between investigated SNVs in rs7093069 and the risk of MS development.

We have also observed significant differences between healthy control subjects and individuals with MS in the allele and genotype frequencies in rs3087243 of the CTLA-4 gene. The G allele, as well as the GG and GA genotypes, was significantly more common among healthy control participants than in subjects with MS. Interestingly, the GG genotype has been identified only in the group of healthy subjects. The CTLA-4 gene has been associated with susceptibility to MS also by Kantarci et al. in the North American MS genomic screen population [32]. However, a study by Rasmussen $\mathrm{HB}$ et al. did not show any interaction between CTLA4 genetic variants that could be involved in the development of MS among European Caucasians [33]. A study by other authors suggests that the involvement of the CTLA4 gene in the pathogenesis of MS may be very subtle, and, rather than genetic polymorphisms that may predispose to MS development, it seems to be related to the functional changes in its pathway [34]. The discrepancies between observations may be due to the differences between studied populations 
TABLE 2: The genotypes and allele frequencies in the studied groups.

\begin{tabular}{|c|c|c|c|c|c|c|c|c|}
\hline \multirow{2}{*}{ Gene } & \multirow{2}{*}{ SNV } & \multirow{2}{*}{ Genotypes/allele } & \multicolumn{2}{|c|}{ MS $(n=94)$} & \multicolumn{2}{|c|}{$\mathrm{HC}(n=160)$} & \multirow[b]{2}{*}{$p *$} & \multirow{2}{*}{ OR $(95 \% \mathrm{CI})$} \\
\hline & & & $n$ & Frequency & $n$ & Frequency & & \\
\hline \multirow{10}{*}{ IL2RA } & rs7093069 & $\mathrm{CC}$ & 57 & $(60.64 \%)$ & 69 & $(43.12 \%)$ & NS & \\
\hline & \multirow{9}{*}{ rs12722598 } & CT & 36 & $(38.8 \%)$ & 83 & $(51.88 \%)$ & 0.016 & 0.52 \\
\hline & & TT & 1 & $(1.06 \%)$ & 8 & $(5.0 \%)$ & 0.047 & 0.15 \\
\hline & & $\mathrm{C}$ & 150 & $(0.80)$ & 221 & $(0.69)$ & \multirow{2}{*}{0.0053} & \multirow{2}{*}{0.56} \\
\hline & & $\mathrm{T}$ & 38 & $(0.20)$ & 99 & $(0.31)$ & & \\
\hline & & TT & 80 & $(85.11 \%)$ & 156 & $(97.5 \%)$ & NS & \multirow{3}{*}{4.2} \\
\hline & & $\mathrm{TC}$ & 14 & $(14.89 \%)$ & 3 & $(1.88 \%)$ & 0.06 & \\
\hline & & CC & - & & 1 & $(0.62 \%)$ & NS & \\
\hline & & $\mathrm{T}$ & 174 & $(0.93)$ & 315 & $(0.99)$ & \multirow{2}{*}{0.001} & \multirow{2}{*}{0.2} \\
\hline & & $\mathrm{C}$ & 14 & $(0.07)$ & 4 & $(0.01)$ & & \\
\hline \multirow{15}{*}{ CTLA4 } & \multirow[t]{5}{*}{ rs3087243 } & GG & 0 & $(0.00 \%)$ & 69 & $(43.12 \%)$ & 0.00 & 0.0038 \\
\hline & & GA & 30 & $(31.91 \%)$ & 77 & $(48.12 \%)$ & 0.00 & \multirow[t]{4}{*}{0.087} \\
\hline & & AA & 64 & $(68.09 \%)$ & 14 & $(8.75 \%)$ & NS & \\
\hline & & G & 30 & $(0.16)$ & 215 & $(0.67)$ & \multirow{2}{*}{0.00} & \\
\hline & & A & 158 & $(0.84)$ & 105 & $(0.33)$ & & \\
\hline & \multirow[t]{5}{*}{ rs231775 } & AA & 36 & $(38.30 \%)$ & 51 & $(31.88 \%)$ & \multicolumn{2}{|l|}{ NS } \\
\hline & & AG & 45 & $(47.87 \%)$ & 82 & $(51.25 \%)$ & NS & 0.78 \\
\hline & & GG & 13 & $(13.83 \%)$ & 27 & $(16.88 \%)$ & NS & \multirow[t]{3}{*}{0.68} \\
\hline & & A & 117 & $(0.62)$ & 184 & $(0.58)$ & \multirow{2}{*}{ NS } & \\
\hline & & G & 71 & $(0.38)$ & 136 & $(0.43)$ & & \\
\hline & \multirow[t]{5}{*}{ rs5742906 } & CC & 71 & $(75.53 \%)$ & 109 & $(68.13 \%)$ & NS & \\
\hline & & CT & 20 & $(21.28 \%)$ & 40 & $(25.00 \%)$ & NS & 0.77 \\
\hline & & $\mathrm{TT}$ & 3 & $(3.19 \%)$ & 11 & $(6.88 \%)$ & NS & 0.41 \\
\hline & & $\mathrm{C}$ & 162 & $(0.86)$ & 258 & $(0.81)$ & \multirow{2}{*}{0.069} & \\
\hline & & $\mathrm{T}$ & 26 & $(0.14)$ & 62 & $(0.19)$ & & \\
\hline \multirow{5}{*}{ CD40 } & \multirow[t]{5}{*}{ rs1883832 } & TT & 7 & $(7.45 \%)$ & 10 & $(6.25 \%)$ & NS & \\
\hline & & TC & 23 & $(24.47 \%)$ & 59 & $(36.88 \%)$ & 0.0477 & 0.55 \\
\hline & & CC & 64 & $(68.09 \%)$ & 91 & $(56.88 \%)$ & NS & 0.99 \\
\hline & & $\mathrm{T}$ & 37 & $(0.20)$ & 79 & $(0.25)$ & NS & \\
\hline & & $\mathrm{C}$ & 151 & $(0.80)$ & 241 & $(0.75)$ & 1vo & \\
\hline & rs1748033 & $\mathrm{TT}$ & 7 & $(7.45 \%)$ & 13 & $(8.13 \%)$ & NS & \\
\hline & & $\mathrm{TC}$ & 45 & $(47.87 \%)$ & 58 & $(36.25 \%)$ & 0.068 & 1.64 \\
\hline PADI4 & & CC & 42 & $(44.68 \%)$ & 89 & $(55.63 \%)$ & NS & 1.14 \\
\hline & & $\mathrm{T}$ & 59 & $(0.31)$ & 84 & $(0.26)$ & IS & \\
\hline & & $\mathrm{C}$ & 129 & $(0.69)$ & 236 & $(0.74)$ & No & \\
\hline
\end{tabular}

*For genotypes, we report $p$ value for $\mathrm{Chi}^{2}$ test, and for alleles, we report Fisher's exact $p$ value. SNV, single-nucleotide variant; MS, multiple sclerosis; HC, healthy control; OR, odds ratio.

and require further investigation, especially since in our studied group the GG genotype has been identified only in healthy subjects, which is intriguing. Nevertheless, when comparing results, the ethnic differences between studied populations should be always considered, as for example in our previous study, we did not observe any associations between the MTHFR mutation and the development of MS in the Polish population [35], which has been reported in the different populations [36].

Our analysis of the rs1883832 polymorphisms in the CD40 gene has shown that only the heterozygous TC variant is more common among healthy individuals compared to subjects with MS, without any differences between homozygous variant frequencies. Although the T allele of rs1883832 has been previously associated with increased susceptibility to MS [22], MS development, age of onset, and progression, including in the Polish population [37], we did not observe this association in our studied group. This could be due to the small study sample in our analysis, but also the regional differences cannot be excluded. Moreover, the importance and role of the CD40 gene variants in MS development is not clear and still under investigation. A lower expression of 
CD40, being a costimulator of T-cell activation, has been associated with increased risk of MS development, despite that the same $\mathrm{CD} 40$ variant may be associated with the reduced risk of other inflammatory ADs, and could be consistent with other, for example immunoregulatory, functions of CD40, playing an important role in protection from MS development [38].

In our study population, we did not notice any significant differences between cases and control group in the frequencies of alleles and genotypes of rs1748033 in the PADI4 gene. Our results are in line with the study by Tommasi et al. [39]. The role of PADI4 isoforms in some ADs has been studied extensively, and even if in MS the extensive deamination of brain proteins is observed in active lesions, the role of the PADI4 gene in susceptibility to MS development has not been described so far [40].

Also, we did not notice any crucial differences between studied genotypes in the clinical manifestations of MS that could be explained by the limited samples described herein. Moreover, the cerebral activity and connectivity of neural circuits responsible for locomotor functions, that are included in the EDSS scoring, depend also on the other factors that we have not investigated in our study, such as serum concentrations of vitamin D [41], which is involved in the immune responses and brain functions [42] and is considered a serum biomarker in neurological diseases as well [43]. The risk, progression, and clinical manifestation of MS has been shown to be associated also with the other SNVs [44] that we have not analysed in our study and might have an impact on our results. Nevertheless, the small study sample is a major limitation of our study; therefore, our results should be interpreted with caution, and further investigations and observations in the larger population are required.

\section{Conclusions}

In summary, despite the relatively small study group, our results show that some of the investigated SNVs within the IL2RA, CTAL4, and CD40 genes are more common in MS individuals, and others may have a protective role, indicating the need and importance of further investigation.

\section{Data Availability}

The data used to support the findings of this study are available from the corresponding author upon request.

\section{Conflicts of Interest}

The authors declare that they have no competing interests.

\section{Authors' Contributions}

MC is responsible for conceptualisation, methodology, investigation, and writing-original draft preparation. NWK is responsible for methodology and investigation. EAP is responsible for writing - original draft preparation. $\mathrm{OZ}$ is responsible for methodology and statistical analysis. KKT and RP are responsible for investigation. $\mathrm{AJK}, \mathrm{JK}$, and $\mathrm{AK}$ is responsible for supervision. Authors read and approved the final manuscript.

\section{Acknowledgments}

We thank the Medical University of Bialystok staff for their contributions and help with data collection and laboratory analysis.

\section{References}

[1] P. S. Ramos, A. M. Shedlock, and C. D. Langefeld, "Genetics of autoimmune diseases: insights from population genetics," Journal of Human Genetics, vol. 60, no. 11, pp. 657-664, 2015.

[2] A. Lleo, P. Invernizzi, B. Gao, M. Podda, and M. E. Gershwin, "Definition of human autoimmunity-autoantibodies versus autoimmune disease," Autoimmunity Reviews, vol. 9, no. 5, pp. A259-A266, 2010.

[3] Y. Kochi, "Genetics of autoimmune diseases: perspectives from genome-wide association studies," International Immunology, vol. 28, no. 4, pp. 155-161, 2016.

[4] M. Gutierrez-Arcelus, S. S. Rich, and S. Raychaudhuri, "Autoimmune diseases - connecting risk alleles with molecular traits of the immune system," Nature Reviews. Genetics, vol. 17, no. 3, pp. 160-174, 2016.

[5] D. P. Singal and M. A. Blajchman, "Histocompatibility (HL-A) antigens, lymphocytotoxic antibodies and tissue antibodies in patients with diabetes mellitus," Diabetes, vol. 22, no. 6, pp. 429-432, 1973.

[6] P. Stastny, "Mixed lymphocyte cultures in rheumatoid arthritis," The Journal of Clinical Investigation, vol. 57, no. 5, pp. 1148-1157, 1976.

[7] K. L. Moser, J. A. Kelly, C. J. Lessard, and J. B. Harley, "Recent insights into the genetic basis of systemic lupus erythematosus," Genes and Immunity, vol. 10, no. 5, pp. 373-379, 2009.

[8] J. R. Oksenberg, L. F. Barcellos, B. A. Cree et al., "Mapping multiple sclerosis susceptibility to the HLA-DR locus in African Americans," American Journal of Human Genetics, vol. 74, no. 1, pp. 160-167, 2004.

[9] J. A. Noble, A. Martin, A. M. Valdes et al., “Type 1 diabetes risk for human leukocyte antigen (HLA)-DR3 haplotypes depends on genotypic context: association of DPB1 and HLA class I loci among DR3- and DR4-matched Italian patients and controls," Human Immunology, vol. 69, no. 4-5, pp. 291-300, 2008.

[10] S. C. Gough, L. S. Walker, and D. M. Sansom, "CTLA4 gene polymorphism and autoimmunity," Immunological Reviews, vol. 204, no. 1, pp. 102-115, 2005.

[11] J.-E. Martin, and Spanish Scleroderma Group, F. D. Carmona et al., "The autoimmune disease-associated IL2RA locus is involved in the clinical manifestations of systemic sclerosis," Genes and Immunity, vol. 13, no. 2, pp. 191-196, 2012.

[12] D. J. Rawlings, X. Dai, and J. H. Buckner, "The role of PTPN22 risk variant in the development of autoimmunity: finding common ground between mouse and human," Journal of Immunology, vol. 194, no. 7, pp. 2977-2984, 2015.

[13] N. Wawrusiewicz-Kurylonek, O. M. Koper-Lenkiewicz, J. Gościk, J. Myśliwiec, P. Pawłowski, and A. J. Krętowski, "Association of PTPN22 polymorphism and its correlation with Graves' disease susceptibility in Polish adult populationa preliminary study," Molecular Genetics \& Genomic Medicine, vol. 7, no. 6, article e661, 2019. 
[14] C. Baecher-Allan, B. J. Kaskow, and H. L. Weiner, "Multiple sclerosis: mechanisms and immunotherapy," Neuron, vol. 97, no. 4, pp. 742-768, 2018.

[15] International Multiple Sclerosis Genetics Consortium (IMSGC), "Analysis of immune-related loci identifies 48 new susceptibility variants for multiple sclerosis," Nature Genetics, vol. 45, no. 11, pp. 1353-1360, 2013.

[16] W. A. Teft, M. G. Kirchhof, and J. Madrenas, "A molecular perspective of CTLA-4 function," Annual Review of Immunology, vol. 24, no. 1, pp. 65-97, 2006.

[17] L. M. Maier, D. E. Anderson, P. L. De Jager, L. S. Wicker, and D. A. Hafler, "Allelic variant in CTLA4 alters T cell phosphorylation patterns," Proceedings of the National Academy of Sciences of the United States of America, vol. 104, no. 47, pp. 18607-18612, 2007.

[18] K. H. Toomer, J. B. Lui, N. H. Altman, Y. Ban, X. Chen, and T. R. Malek, "Essential and non-overlapping IL-2R $\alpha$-dependent processes for thymic development and peripheral homeostasis of regulatory T cells," Nature Communications, vol. 10, no. 1, p. 1037, 2019.

[19] T. R. Malek, "T helper cells, IL-2 and the generation of cytotoxic T-cell responses," Trends in Immunology, vol. 23, no. 10, pp. 465-467, 2002.

[20] S. Buhelt, H. B. Søndergaard, A. Oturai, H. Ullum, M. R. von Essen, and F. Sellebjerg, "Relationship between multiple sclerosis-associated IL2RA Risk Allele Variants and Circulating $\mathrm{T}$ Cell Phenotypes in Healthy Genotype-Selected Controls," Cells, vol. 8, no. 6, p. 634, 2019.

[21] M. E. Munroe and G. A. Bishop, "A costimulatory function for T cell CD40," Journal of Immunology, vol. 178, no. 2, pp. 671682, 2007.

[22] J. Qin, J. Xing, R. Liu, B. Chen, Y. Chen, and X. Zhuang, “Association between CD40 rs1883832 and immune-related diseases susceptibility: a meta-analysis," Oncotarget, vol. 8, no. 60, pp. 102235-102243, 2017.

[23] D. S. Korobko, N. A. Malkova, E. V. Bulatova et al., "The effect of genetic factors on the phenotypic expression of multiple sclerosis," Zhurnal Nevrologii i Psikhiatrii Imeni S.S. Korsakova, vol. 113, pp. 10-16, 2013.

[24] X. Chang, R. Yamada, A. Suzuki et al., "Localization of peptidylarginine deiminase 4 (PADI4) and citrullinated protein in synovial tissue of rheumatoid arthritis," Rheumatology (Oxford, England), vol. 44, no. 1, pp. 40-50, 2005.

[25] Y. Liu, Y. L. Lightfoot, N. Seto et al., "Peptidylarginine deiminases 2 and 4 modulate innate and adaptive immune responses in TLR-7-dependent lupus," JCI Insight, vol. 3, no. 23, 2018.

[26] A. L. Zozulya and H. Wiendl, "The role of regulatory T cells in multiple sclerosis," Nature Clinical Practice. Neurology, vol. 4, no. 7, pp. 384-398, 2008.

[27] M. Chorąży, N. Wawrusiewicz-Kurylonek, R. Posmyk et al., "Analysis of chosen SNVs in GPC5, CD58 and IRF8 genes in multiple sclerosis patients," Advances in Medical Sciences, vol. 64, no. 2, pp. 230-234, 2019.

[28] A. Czarnowska, K. Kapica-Topczewska, O. Zajkowska et al., "Herpesviridae seropositivity in patients with multiple sclerosis: first Polish study," European Neurology, vol. 80, no. 5-6, pp. 229-235, 2019.

[29] P. A. Gourraud, H. F. Harbo, S. L. Hauser, and S. E. Baranzini, "The genetics of multiple sclerosis: an up-to-date review," Immunological Reviews, vol. 248, no. 1, pp. 87-103, 2012.
[30] F. J. Hartmann, M. Khademi, J. Aram et al., "Multiple sclerosis-associated IL2RA polymorphism controls GM-CSF production in human TH cells," Nature Communications, vol. 5, no. 1, p. 5056, 2014.

[31] S. Sawcer, G. Hellenthal, M. Pirinen, C. C. Spencer, N. A. Patsopoulos, L. Moutsianas et al., "Genetic risk and a primary role for cell-mediated immune mechanisms in multiple sclerosis," Nature, vol. 476, no. 7359, pp. 214-219, 2011.

[32] O. H. Kantarci, D. D. Hebrink, S. J. Achenbach et al., "CTLA4 is associated with susceptibility to multiple sclerosis," Journal of Neuroimmunology, vol. 134, no. 1-2, pp. 133-141, 2003.

[33] H. B. Rasmussen, M. A. Kelly, D. A. Francis, and J. Clausen, "CTLA4 in multiple sclerosis. Lack of genetic association in a European Caucasian population but evidence of interaction with HLA-DR2 among Shanghai Chinese," Journal of the Neurological Sciences, vol. 184, no. 2, pp. 143-147, 2001.

[34] R. Palacios, D. Comas, J. Elorza, and P. Villoslada, "Genomic regulation of CTLA4 and multiple sclerosis," Journal of Neuroimmunology, vol. 203, no. 1, pp. 108-115, 2008.

[35] M. Chorąży, N. Wawrusiewicz-Kurylonek, J. Gościk et al., "Association between polymorphisms of a folate - homocysteine - methionine - SAM metabolising enzyme gene and multiple sclerosis in a Polish population," Neurologia $i$ Neurochirurgia Polska, vol. 53, no. 3, pp. 194-198, 2019.

[36] L. Klotz, M. Farkas, N. Bain et al., "The variant methylenetetrahydrofolate reductase c.1298A $>$ C (p.E429A) is associated with multiple sclerosis in a German case-control study," Neuroscience Letters, vol. 468, no. 3, pp. 183-185, 2010.

[37] M. Wagner, A. Wisniewski, M. Bilinska et al., "Investigation of gene-gene interactions between CD40 and CD40L in Polish multiple sclerosis patients," Human Immunology, vol. 75, no. 8, pp. 796-801, 2014.

[38] J. Field, F. Shahijanian, S. Schibeci et al., "The MS risk allele of CD40 is associated with reduced cell-membrane bound expression in antigen presenting cells: implications for gene function," PLoS One, vol. 10, no. 6, article e0127080, 2015.

[39] C. Tommasi, E. Petit-Teixeira, I. Cournu-Rebeix et al., "PADI4 gene in multiple sclerosis: a family-based association study," Journal of Neuroimmunology, vol. 177, no. 1-2, pp. 142-145, 2006.

[40] C. Anzilotti, F. Pratesi, C. Tommasi, and P. Migliorini, "Peptidylarginine deiminase 4 and citrullination in health and disease," Autoimmunity Reviews, vol. 9, no. 3, pp. 158-160, 2010.

[41] G. Bivona, C. M. Gambino, G. Iacolino, and M. Ciaccio, "Vitamin D and the nervous system," Neurological Research, vol. 41, no. 9, pp. 827-835, 2019.

[42] G. Bivona, L. Agnello, C. Bellia et al., "Non-skeletal activities of vitamin D: from physiology to brain pathology," Medicina (Kaunas, Lithuania), vol. 55, no. 7, p. 341, 2019.

[43] G. Bivona, B. Lo Sasso, G. Iacolino et al., "Standardized measurement of circulating vitamin $\mathrm{D}[25(\mathrm{OH}) \mathrm{D}]$ and its putative role as a serum biomarker in Alzheimer's disease and Parkinson's disease," Clinica Chimica Acta, vol. 497, pp. 82-87, 2019.

[44] C. Scazzone, L. Agnello, P. Ragonese et al., "Association of CYP2R1 rs10766197 with MS risk and disease progression," Journal of Neuroscience Research, vol. 96, no. 2, pp. 297-304, 2018. 